\title{
Leptospirose beim Hund - ein Update
}

Thierry Francey, Ariane Schweighauser

Die Leptospirose ist eine der wichtigsten Zoonosen weltweit. Die Krankheit kann durch über 250 pathogene Serovare von Leptospira interrogans verursacht werden, was ihre Diagnose und Prophylaxe erheblich erschweren kann.

Klinisch manifestiert sich die Infektion beim Hund in den meisten Fällen als Nierenversagen, z.T. auch als Leberversagen, Lungenblutungen oder selten als systemisches hämorrhagisches Syndrom. Die einzelnen Manifestationen variieren individuell sehr stark im Schweregrad, von subklinischer Läsion bis hin zum multiplen Organversagen. Obwohl die Infektion v.a. auch beim Menschen typischerweise in tropischen und subtropischen Ländern auftritt, ist die canine Leptospirose in unseren Breitengraden weit verbreitet und gilt als Klassiker unter den Hundekrankheiten, mit in den letzten Jahren wieder steigender Prävalenz. Fundierte Kenntnisse ihrer Biologie sind wichtig für eine frühzeitige korrekte Diagnose und somit für einen guten Behandlungserfolg.

\section{Epidemiologie und Pathogenese}

Leptospirose ist eine bakterielle Zoonose, verursacht durch gram-negative spiralförmige Spirochäten der Gattung Leptospira. Sie ist eine der wichtigsten Zoonosen weltweit mit höchster Prävalenz beim Menschen in Entwicklungsländern in tropischen oder subtropischen Gegenden. Sie kommt aber auch in Industrieländern vor, wo ihr Auftreten zudem nicht mehr nur auf ländliche Gebiete begrenzt ist. Weltweit wird ihre Inzidenz auf 1,7 Millionen schwere Fälle und 120000 Todesfälle pro Jahr geschätzt.
Historisch bedingt werden Leptospiren aufgrund ihrer Seroreaktivität in verschiedene Serovare eingeteilt. Diese Antikörperinduktion basiert auf unterschiedlichen Kohlenhydratbestandteilen der Oberflächen-Lipopolysaccharide, sodass bisher über 250 pathogene Serovare identifiziert wurden. Antigenetisch verwandte Serovare mit Kreuzreaktion bei serologischen Tests werden in Serogruppen eingeteilt. Neuere, auf einer genetischen Typisierung der Stämme basierende Einteilungssysteme wurden vorgeschlagen, um die manchmal verwirrende Klassifikation in Serovare zu ersetzen. Diese Systeme haben sich jedoch noch nicht wirklich durchgesetzt.

Leptospiren überleben in der Umwelt entweder frei in kontaminiertem Gewässer und feuchten Gebieten oder als persistierende asymptomatische Infektion in Trägertieren. In unseren Breitengraden stellt das Trägertum in Wirtstieren einen wichtigen Mechanismus für das Überleben der Bakterien in den kalten Wintermonaten dar, da die der Witterung ausgesetzten Leptospiren bei Temperaturen unter dem Gefrierpunkt ebenso schlecht überleben wie unter trocken-heißen Umständen. Unter günstigen Bedingungen - warm und feucht können die Erreger allerdings monatelang in der Umwelt überleben.

Empfängliche Wirtstiere infizieren sich v.a. in der Zeit von Frühling bis Herbst:

- vorwiegend indirekt über kontaminierte stehende Gewässer oder verseuchtes Futter

- seltener durch direkten Kontakt mit Trägertieren

Die Bakterien treten über Schleimhäute und Hautläsionen in den Körper ein. Eine transplazentare Übertragung oder eine Ansteckung über die Muttermilch wurde beim Menschen beschrieben. Die Hauptüberträger in unseren Regionen scheinen

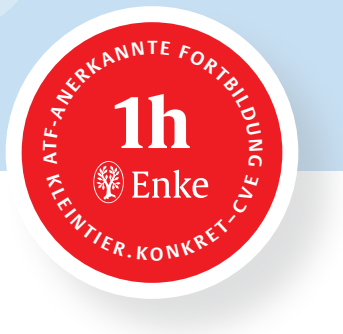

wildlebende Nagetiere (u.a. Ratten und Mäuse) zu sein, die Rolle anderer Wildtiere (z.B. Reh, Hirsch, Dachs, Fuchs und Biber) in der aktuellen Epidemiologie ist noch unklar. Domestizierte Wiederkäuer können ebenfalls als Träger bzw. Ausscheider bedeutend sein, weshalb diese Tierarten in vielen Ländern seuchenpolizeilich überwacht werden.

In mehreren Studien wurden deutlich höhere Prävalenzen bei unkastrierten Rüden als bei Hündinnen beobachtet, was oft durch die erhöhte Exposition der Rüden gegenüber Risikofaktoren in der Umwelt (Harnlecken etc.) erklärt wird. Eine ähnliche Prädisposition bei Männern gegenüber Frauen wurde auch in der Humanmedizin berichtet und es wird über eine mögliche Rolle hormoneller Faktoren diskutiert.

\section{Klinik}

Zu den wichtigsten klinischen Manifestationen der Leptospirose beim Hund gehören:

- akutes Nierenversagen

- Hepatopathie

- Lungenblutungen

- Gerinnungsstörungen

Die Zusammensetzung der verschiedenen Organbeteiligungen ist individuell verschieden und der Schweregrad der Erkrankung variiert von leichtgradiger subklinischer Läsion bis hin zum lebensbedrohlichen multiplen Organversagen. Interessanterweise können sowohl das Spektrum an Organmanifestationen wie auch der Schweregrad der Erkrankung geografisch und von Jahr zu Jahr sehr variabel sein. So prädominiert z.B. an einem Ort oder zu einer gewissen Zeit die Lebermanifestation, während in einer anderen Region oder zu einem anderen 


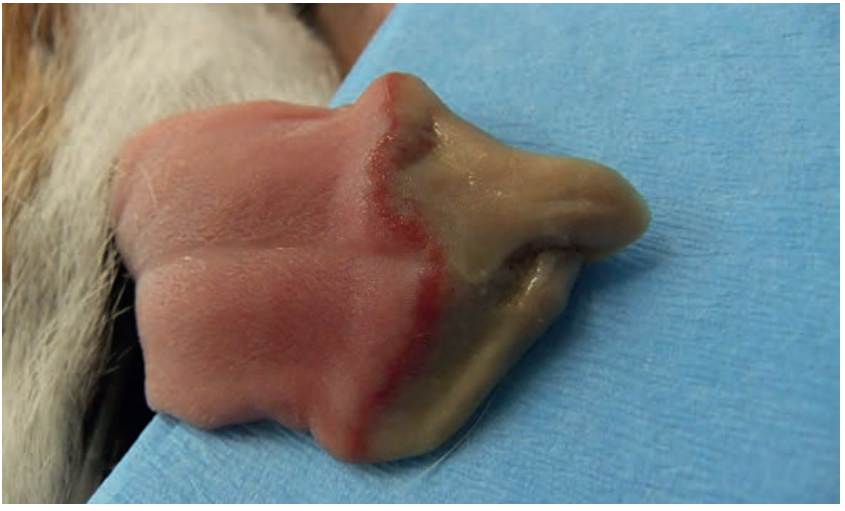

\section{Abb. 1}

Jack Russell Terrier, weiblich kastriert, 8 Jahre alt: Urämische nekrotisierende Glossitis bei einer akuten Leptospirose. Die Zungenspitze musste chirurgisch debridiert und exzidiert werden.
Zeitpunkt die meisten betroffenen Hunde eine anikterische Form zeigen.

Eine der häufigsten Manifestationen der caninen Leptospirose ist zurzeit der akute Niereninsult. Die Infektion führt zu einer interstitiellen Nephritis mit lympho-plasmazellulärer Infiltration. Die Leptospiren sind mit Spezialfärbungen (z.B. Silberimprägnation) im Interstitium oder intrazellulär im Nierenepithel erkennbar. Klinisch werden Hinweise auf einen tubulointerstitiellen Schaden gefunden:

- Polyurie oder Oligoanurie

- Isosthenurie

- renale Glukosurie

- tubuläre/(glomeruläre) Proteinurie

- Zylindrurie

- Azotämie ( $\triangleright$ Abb. 1)

- Hypo- oder Hyperkaliämie

- Hyperphosphatämie

- metabolische Azidose mit hoher Anionenlücke

- systemische Hypertension
Während der Mensch meistens Polyurie zeigt, ist die Leptospirose beim Hund oft mit einer Phase von Oligoanurie verbunden. Letztere kann in der Erholungsphase der Nieren in eine z.T. hochgradige Polyurie übergehen, die oft zu ausgeprägten Natrium- und Kaliumverlusten führt.

Eine Leberbeteiligung bei Leptospirose ist in der Regel durch Ikterus ( $\triangleright$ Abb.2) und Erhöhung der Leberenzyme - v.a. der alkalischen Phosphatase - charakterisiert. Klinisch äußert sich dies vorwiegend durch eine gastrointestinale Symptomatik, seltener durch ein hypoglykämisches Leberversagen. Typische Veränderungen im Ultraschall sind ein Gallenblasenwandödem, eine Mukozele bis hin zur Ruptur der Gallenblase.

Hämorrhagische Formen manifestieren sich überwiegend als disseminierte intravaskuläre Gerinnung (DIG) mit generalisierten Blutungen. Diese Form entspricht der klassischen historischen Be- schreibung, jedoch wurde vor kurzem beobachtet, dass Hämostasestörungen sowohl durch Hypokoagulabilität als auch durch Hyperkoagulabilität (prothrombotischer Zustand) gekennzeichnet sein können. Eine individuelle Abklärung des Hämostasestatus ist beim Verdacht auf eine Leptospirose daher unentbehrlich.

Leptospirose-assoziierte Lungenblutungen treten in gewissen Gebieten sehr häufig auf. In der Schweiz zeigen z.B. über $80 \%$ der Hunde bei der Vorstellung typische Röntgenveränderungen mit interstitiellen bis alveolären, fokalen bis konfluierenden generalisierten Verschattungen ( $\triangleright$ Abb.3). In Deutschland scheint die Lungenbeteiligung geografisch punktuell vorzukommen, mit einer Hochburg z.B. in Berlin und Umgebung. Hunde mit schwerer Lungenbeteiligung weisen eine hohe Mortalitätsrate von über 50\% auf. Obwohl der genaue Mechanismus der Lungenhämorrhagien noch nicht völlig abgeklärt ist, scheinen hochgradige Permeabilitätsstörungen des Lungenepithels via Immunmechanismen oder durch die direkte Wirkung bakterieller Komponenten zu entstehen. Die resultierenden Blutungen im Alveolarraum können sehr schnell dramatisch verlaufen und viele Tiere sterben an akutem Lungenversagen.

\section{Diagnosestellung}

Bei Symptomen wie akutem Nierenversagen, Ikterus, DIG oder Lungen-

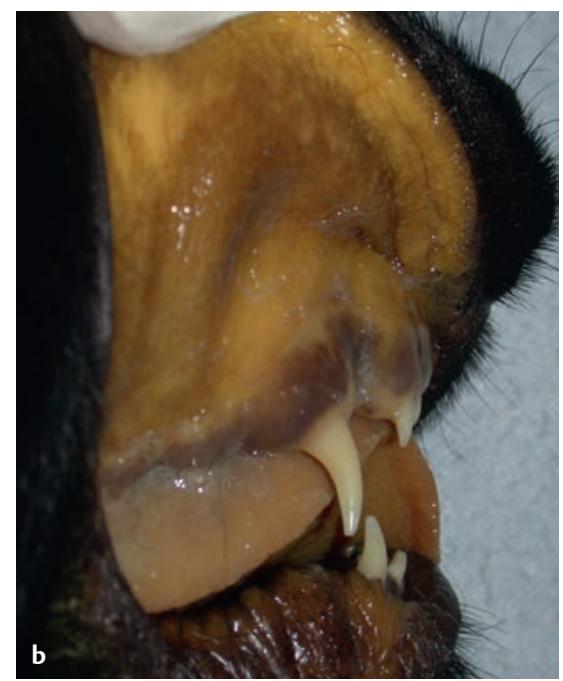

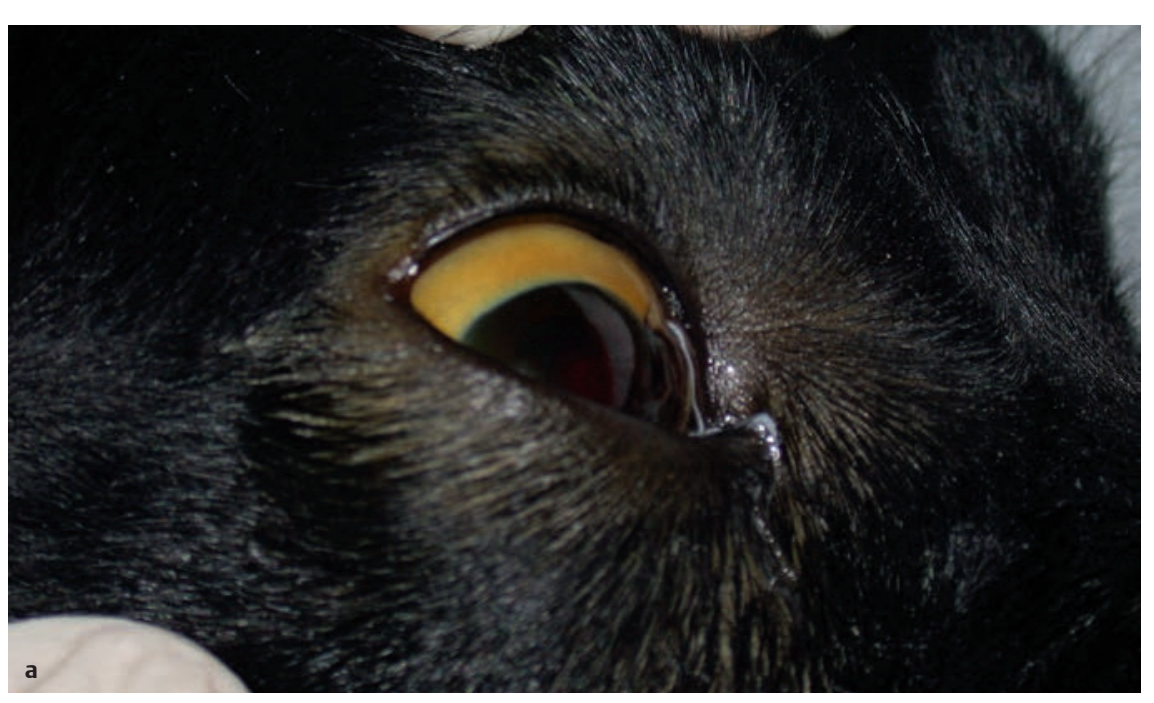

Abb. 2 Flat-Coated Retriever, männlich, 3 Monate alt: Ikterus der (a) Skleren und der (b) Maulschleimhaut bei einer Leptospirose. 


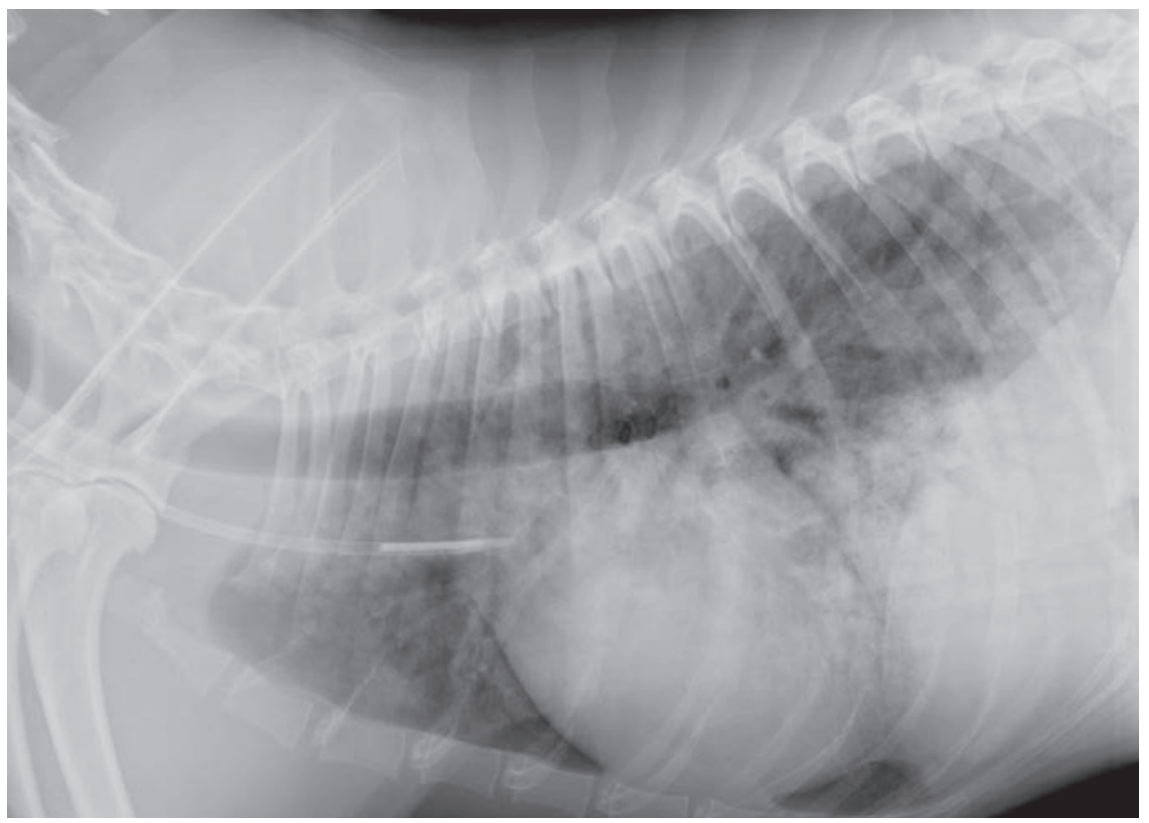

Abb. 3 Mischling, männlich, 4 Jahre alt: Thoraxröntgen mit dem typischen Bild der leptospiroseassoziierten Lungenblutungen.

blutungen unklarer Ätiologie sollte immer an Leptospirose gedacht und dahingehend abgeklärt werden. Diagnostische Tests für Leptospirose beinhalten:

- Serologie (Antikörpernachweis)

- Nachweis von Leptospiren-DNA

- bakteriologische Kulturen

- mikroskopischer Nachweis von Leptospiren

Trotz klarer Limitationen bleibt der Referenztest für die Diagnosestellung der Leptospirose der Mikroagglutinationstest (MAT) mit gepaarten Serumproben, die im Abstand von 2-3 Wochen entnommen werden. Ein 4facher Titeranstieg spricht für einen aktiven Prozess. In einer prospektiven Studie aus einem Endemiegebiet wurde gezeigt, dass ein Antikörpertiter von $\geq 1: 800$ bei der Vorstellung eine Spezifizität von $100 \%$, eine Sensitivität von 50\% und eine Genauigkeit von 64\% aufweist. Somit kann ein erster positiver Titer schon klare Hinweise auf die Ätiologie eines akuten Nierenversagens geben und einen hohen Verdacht aufkommen lassen. Die Hälfte der Hunde mit Leptospirose hat somit allerdings noch ein negatives Testresultat bei der initialen Vorstellung, weshalb eine Verdachtsdiagnose zu diesem Zeitpunkt sicher noch nicht ausgeschlossen werden kann. Da Hunde nach einer Impfung ebenfalls hohe Titer aufweisen können, sollte stets eine gepaarte Serologie durchgeführt werden, um die Diagnose bestätigen zu können.

Um rascher eine Antwort zu bekommen als mittels MAT und um zeitaufwendige Kulturen von lebenden Leptospiren zu umgehen, wurden andere serologische Tests entwickelt.

Schnelltests (z.B. IgM-ELISA) oder konventionelle serologische Tests (IgMund IgG-Nachweis) können erste Hinweise auf die Wahrscheinlichkeit einer Leptospirose liefern und dadurch bei klinischen Entscheidungen helfen. Sie erlauben allerdings keine Zuordnung zu den kausalen Serogruppen, was v.a. für epidemiologische Zwecke von Nachteil ist. Schnelltests sollten immer mit konventioneller MAT-Serologie bestätigt werden, da diese nach wie vor den Goldstandard darstellt und nur die Serokonversion eine aktive Infektion beweisen kann. Gleiche Limitationen für vor kurzem geimpfte Tiere wie bei einer einmalig durchgeführten MAT gilt es nämlich auch bei Schnelltests zu beachten.

Bis vor kurzem gab es in Europa nur bivalente Impfstoffe und die meisten Hunde waren an anderen (nicht im Impfstoff enthaltenen) Serovaren erkrankt. Mit der Einführung von quadrivalenten Impfstoffen, die zusätzlich mit den momentan am häufigsten festgestellten krankheitsverursachenden Serovaren aufge- stockt wurden, ist die Diagnosestellung mittels Serologie deutlich schwieriger geworden. Eine Serokonversion auf ein Serovar, das im Impfstoff enthalten ist, kann entweder ein Versagen des Impfschutzes oder eine normale serologische Impfantwort bedeuten. Bei der Interpretation ist es darum wichtig, den Zeitpunkt der Probenentnahmen genau zu beachten, mit Doppelserologien zu arbeiten und wenn möglich die Diagnose mit PCR zu unterstützen.

Der direkte Leptospiren-Nachweis kann mittels PCR erfolgen. In einer ersten Phase der Krankheit werden Leptospiren nur im Blut gefunden und erst später im Harn. Da dieser Wechsel ungefähr zur Zeit der Vorstellung geschieht, sollten idealerweise beide Medien zur Untersuchung geschickt werden. Wie bei allen PCR-Untersuchungen gilt auch hier Vorsicht bei der Interpretation. Positive und negative Kontrollproben sind wichtig für die Qualitätskontrolle. Geringste Kontaminationen mit Leptospiren-DNA bei der Probenentnahme oder im Labor können schon eine positive Reaktion hervorrufen. Eine weitere Schwierigkeit bei der Leptospirose ist das schnelle Verschwinden von jeglicher Spur von LeptospirenDNA aus Blut und Urin, sobald mit der Antibiotikatherapie begonnen wurde. Dies steht im Gegensatz zu vielen anderen Erregern, deren DNA noch lange nachgewiesen werden kann. Aus Nierengewebe scheint die Leptospiren-DNA hingegen deutlich länger nachweisbar zu sein. Da aber eine Punktion der Niere am lebenden Tier ein invasives Verfahren ist, ist diese Untersuchung eher für eine postmortale Bestätigung gedacht.

Für die genaue Identifizierung von Leptospiren-Stämmen und Serovaren sollte idealerweise eine bakteriologische Kultur durchgeführt werden. Diese Kulturen sind aber sehr empfindlich, wachsen extrem langsam (über Wochen bis Monate) und sind fast unmöglich, wenn das Tier mit Antibiotika vorbehandelt wurde. Somit ist diese Methode nicht praxistauglich.

Ein mikroskopischer Nachweis von Leptospiren kann im Harnsediment mittels Dunkelfeldmikroskopie erfolgen. Diese Methode braucht aber Erfahrung und mindestens $10^{4}$ Leptospiren/ml Probenmaterial, um wahrnehmbar zu sein. Sie 
ist nur bei einem positiven Resultat aussagekräftig und sollte ebenfalls mit konventionellen Methoden bestätigt werden. Daher ist auch dieser Test nicht weit verbreitet im Klinikalltag.

Post mortem können Leptospiren auf histologischen Schnitten nach Silberfärbung mikroskopisch nachgewiesen werden.

\section{konkret}

Sobald eine entsprechende Antibiotikatherapie eingeleitet wird, verschwindet die Leptospiren-DNA aus Blut und Urin, was zu negativen PCR-Ergebnissen auch bei infizierten Tieren führen kann.

\section{Therapie}

Die Therapie der caninen Leptospirose folgt 2 Hauptachsen:

- Antibiotikatherapie

- symptomatische Behandlung der Läsionen bzw. Funktionsbeeinträchtigungen der betroffenen Organe und Systeme

Je nach klinischem Schweregrad der Erkrankung sollte eine Überweisung an eine spezialisierte Klinik in Betracht gezogen werden ( $\triangleright$ Tab. 1$)$.

\section{Antibiose}

Die Empfehlungen für die Antibiotikatherapie der Leptospirose wurden zum größten Teil aus experimentellen Daten von anderen Spezies extrapoliert. Ein möglichst früher Therapiestart scheint dabei essenziell für den Erfolg zu sein: Aus diesem Grund sollte eine Therapie bereits auf Verdacht eingeleitet werden, ohne erst die Laborbestätigung abzuwarten. Deshalb ist es wichtig, das klinische Bild gut zu kennen und die Erkrankung bei entsprechender Symptomatik stets auf die Liste der Differenzialdiagnosen zu setzen.

Das Grundantibiotikum für die Behandlung der Leptospirose ist Doxycyclin in einer Dosierung von $5 \mathrm{mg} / \mathrm{kg}$ alle $12 \mathrm{~h}$ oral für 2-3 Wochen. Doxycyclin wirkt sowohl gegen die Bakteriämie wie auch gegen das Trägertum in den Nierentubuli. Allerdings verursachen Tetrazykline oft ausgeprägte gastrointestinale

Tab. 1 Indikationen zur Hospitalisation bei Leptospirose mit spezialisierter Behandlung.

\begin{tabular}{|l|l|}
\hline Formen & Symptomatik \\
\hline renale Form & progressive klinische Verschlechterung trotz adäquater \\
& Therapie \\
- Hyperkaliämie (Kalium $>6$ mmol/l) \\
- Flüssigkeitsüberladung und Hypervolämie \\
- therapieresistente Hypertension \\
\hline - Mukozele der Gallenblase \\
\hline hepatische Form & progressive und hochgradige Hyperbilirubinämie \\
& - Hämostasestörung, die eine Intervention benötigt \\
\hline hämorrhagische Form & Dyspnoe - idealerweise bereits bei nur leichtgradiger \\
\hline pulmonale Form & Symptomatik, da ein Transport zu späterem Zeitpunkt \\
& kritisch bis lebensbedrohlich sein kann \\
\hline
\end{tabular}

Nebenwirkungen, was ihre Anwendung in der akuten Krankheitsphase einschränkt. In diesem Stadium wird daher empfohlen, mit einem besser verträglichen intravenösen Penizillin-Derivat anzufangen, wie z.B. Penizillin G (25000-40000 U/kg alle 6-12 h), Amoxicillin (10-20 mg/kg alle 8-12 h, Clamo$\mathrm{xyl}^{\circledR}$ ) oder Ampicillin-Sulbactan (30 mg/ $\mathrm{kg}$ alle 8-12 h). Penizilline sind hochwirksam gegen die Bakteriämie und somit ausreichend für die akute Phase. Da sie aber keine Wirkung gegen intrazelluläre Persistenz in den Nierentubuli und damit gegen Trägertum und Dauerausscheidung haben, muss die Antibiose zwingend in einer 2. Phase - sobald die Hunde vom Allgemeinzustand her stabil sind - auf Doxycyclin (5 mg/kg alle $12 \mathrm{~h}$ oral für 2 Wochen) umgestellt werden. Dies ist in der Regel erst indiziert, wenn die Patienten kein Erbrechen mehr zeigen und wieder mit Appetit fressen.

Der Einsatz von Fluorochinolonen und Cephalosporinen 3. Generation wurde beschrieben, wird aber nicht als 1 . Wahl empfohlen.

\section{Symptomatische Behandlung}

\section{Akutes Nierenversagen}

Das mit Leptospirose assoziierte akute Nierenversagen ist hauptsächlich durch eine starke interstitielle Entzündung und parenchymatöse Blutungen charakterisiert, während eine Tubulusepithel-Nekrose meistens nicht im Vordergrund steht. Deshalb zeigt diese Form von akutem Nierenversagen eine gute renale Erholungsrate. Solange die Tiere durch die kritische Phase hindurch am Leben erhalten werden können, liegt die Erfolgs- chance für die Nierenerholung zwischen 80-90\%. Ungefähr die Hälfte dieser Hunde zeigt längerfristig eine vollständige Erholung und eine normale Nierenfunktion.

In der initialen Phase kann die Nierenfunktion eingeschränkt bis völlig abwesend sein. Eine konservative medikamentöse Therapie ist daher auf die Patienten beschränkt, die noch genügend Restfunktion haben, um den Organismus vorübergehend und unter vernünftigen Bedingungen am Leben zu erhalten.

Die tägliche Evaluation des Tieres muss daher auch als Ziel haben, den erzielten Erfolg und die Grenzen der momentanen Therapie kritisch zu beurteilen sowie die Notwendigkeit von Blutreinigungsverfahren wie Hämodialyse oder Peritonealdialyse für die Überbrückung dieser sensiblen Phase abzuschätzen. Der Einsatz dieser Therapien bei der Hundeleptospirose hat erlaubt, die Überlebensrate bei Nierenbeteiligung von $40 \%$ auf $90 \%$ zu erhöhen.

Progressive urämische Symptomatologie, persistierende Oligoanurie, mittelbis hochgradige Hyperkaliämie sowie therapieresistente Flüssigkeitsüberladung sind Parameter, die die Grenzen einer konventionellen Therapie anzeigen. Das ganzheitliche klinische Bild ist dabei für diese Entscheidung häufig wichtiger als fixe Laborwerte. Wichtig ist dabei, dass der klinische Verlauf dieser Erkrankung manchmal sehr schnell und auch für den geübten Kliniker unvorhersehbar ist und eine plötzliche unerwartete Verschlechterung jederzeit auftreten kann. Die frühzeitige proaktive 
Abklärung der Verfügbarkeit solcher Nierenersatz-Therapien ist daher grundsätzlich zu empfehlen.

Die Hauptziele der Therapie des akuten Nierenversagens beinhalten folgende Punkte:

- die Restauration physiologischer Hydratationsbedingungen und eines normalen intravaskulären Volumens mittels einer adäquaten Flüssigkeitstherapie. Je nach Fall werden z.B. $\mathrm{NaCl} \mathrm{0,9 \%}$ oder Ringerlaktat eingesetzt. Dabei muss besonders beachtet werden, die Therapie nicht zu aggressiv anzusetzen, da Überhydratation und Hypervolämie sehr viele negative Effekte auf den ganzen Körper haben und mindestens so schädlich wie ein Flüssigkeitsmangel sind.

- die Korrektur von Elektrolyt- und Säuren-Basen-Störungen

- die Korrektur einer systemischen Hypertension mit Amlodipin

- die Behandlung von gastrointestinalen Störungen mit Antiemetika (Maropitant, Ondansetron, Metoclopramid, Chlorpromazin), Magensäurehemmer (Omeprazol, Ranitidin) und eventuell Magenschleimhautschutz (Sucralfat)

- die Unterstützung der Ernährung

- eine angepasste Schmerztherapie

Zwei Klassen von Medikamenten, die in der Regel keinen Platz bei der Behandlung von akutem Nierenversagen haben, sind nicht steroidale Entzündungshemmer und ACE-Hemmer. Erstere führen zu einem erhöhten Risiko von gastrointestinalen und renalen Nebenwirkungen und werden bei diesen Patienten am besten durch Opiate wie Buprenorphin $(0,01 \mathrm{mg} / \mathrm{kg}$ i.v. alle $6-8 \mathrm{~h})$, Methadon (0,1-0,2 $\mathrm{mg} / \mathrm{kg}$ i.v. alle 4-6 h) oder Fentanyl $(2-5 \mu \mathrm{g} / \mathrm{kg} / \mathrm{h}$ Dauertropfinfusion i.v.) ersetzt. Während die ACE-Hemmer in der Behandlung der chronischen stabilen Nierenerkrankung unter entsprechenden Bedingungen und strikter Therapiekontrolle durchaus ihren Platz haben, so

\section{konkret}

Wenn die infizierten Hunde die akute Phase des Nierenversagens überleben, zeigen sie eine sehr gute renale Erholungsrate. kann ihr Einsatz für einen Patienten im akuten Nierenversagen wegen des Risikos von zusätzlicher Hyperkaliämie und der weiteren Verminderung der glomerulären Filtrationsrate durchaus letal enden.

\section{Hepatopathie}

Trotz manchmal ausgeprägtem Ikterus ist es selten, dass die Leberaffektion bei der Leptospirose eine spezifische Therapie verlangt. Antibiose, Flüssigkeitstherapie und symptomatische Behandlung der gastrointestinalen Manifestationen sind in der Regel ausreichend. Die Integrität der Gallenwege sollte aber in jedem Fall kontrolliert und verfolgt werden. Mukozelen werden regelmäßig beobachtet und können zur Ruptur der Gallenblase und biliären Peritonitis führen. Der Einsatz von Antioxidanzien (z.B. S-Adenosyl-Methionin), Choleretika oder weiteren symptomatischen Leberbehandlungen wurde nicht systematisch evaluiert und sollte von Fall zu Fall individuell überdacht werden.

\section{Hämorrhagisches Syndrom}

Tiere mit schwerwiegenden Hämostasestörungen und diffusen Blutungen brauchen eine gründliche diagnostische Aufarbeitung und eine intensive Behandlung, was in der Regel die Überweisung zu einer spezialisierten Klinik bedingt. Nach Störungen der primären Hämostase (Thrombozytopenie und -pathie) oder der sekundären Hämostase (disseminierte intravaskuläre Gerinnung) sowie Hyperkoagulabilität muss aktiv gesucht werden und ihre Aufarbeitung benötigt meistens spezifische Tests. Ihre Behandlung mit Blutprodukten oder mit antithrombotischen Mitteln muss zwingend unter strikter Aufsicht und mit einem entsprechenden Monitoring erfolgen.

\section{Pulmonales hämorrhagisches Syndrom} Dieses Syndrom von Atemnot ist in der Regel unabhängig vom allgemeinen hämorrhagischen Syndrom der Leptospirose. Eine Immunkomponente oder ein direkter Angriff der alveolären Membran durch Bakterienprodukte scheint an der Pathogenese beteiligt zu sein. Leider gibt es momentan noch keine gute Therapie dieser oft fatalen Manifestation und das Hauptgewicht liegt daher auf der Prophylaxe. Faktoren, die zur Progression von beginnender Blutung führen, müssen eliminiert werden, d.h. konkret muss man:

- Aufregung und Stress vermeiden

- systemische Hypertension unter Kontrolle bringen

- Hypervolämie behandeln

Der Einsatz von Immunsuppressoren oder Eingriffe, um die primäre Hämostase mit Desmopressin zu aktivieren, wird zurzeit wegen Mangel an konkreten Daten und potenziellen Risiken nicht allgemein empfohlen.

\section{Zoonotisches Risiko}

Wegen des zoonotischen Risikos der Leptospirose ist es wichtig, sich gegen diese Infektion zu schützen und jeglichen direkten Kontakt der Haut oder der Schleimhäute mit Körperflüssigkeiten des Tieres zu vermeiden. Insbesondere sollte jede Manipulation infizierter Hunde ausschließlich mit Handschuhen erfolgen. Weiterhin empfiehlt sich ein besonderer Schutz mit Maske und Brille in Situationen, in denen Aerosolbildung möglich ist (z.B. Beurteilung der Atemwege, Intubation, Reinigung der Boxen). Die Besitzer sollten ebenfalls auf das Risiko und die klinischen Manifestationen aufmerksam gemacht werden. Grippeähnliche Symptome, Gliederschmerzen und Fieber gehören zu den ersten klinischen Zeichen beim Menschen. Bei Progression kann es auch hier zu Nierenversagen, Lungenblutungen etc. kommen. Mit angemessenen Schutzmaßnahmen und Achtsamkeit ist das zoonotische Risiko der Leptospirose im Rahmen der tierärztlichen Arbeit eher gering allerdings muss dafür zuerst an die Krankheit gedacht werden.

\section{Prophylaxe}

Die beste Vorbeugung gegen Leptospirose in einem Endemiegebiet ist die Impfung. Mit der breitflächigen Einführung der Leptospirose-Impfung als Teil der Core-Impfstoffe in den 1970er Jahren ist diese Infektion in unseren Ländern beim Kleintier fast verschwunden. Die Verlagerung des Spektrums an krank- 
machenden Serovaren in den letzten 10 Jahren zu solchen, die in den früheren Impfstoffen nicht enthalten waren, hat sicher zur beobachteten explosiven $\mathrm{Zu}$ nahme der Fälle beigetragen.

Es ist zu hoffen, dass der neue quadrivalente Impfstoff einen adäquaten Schutz für unsere epidemiologische Situation bringen wird, da das damit abgedeckte Spektrum deutlich geeigneter erscheint. Daten aus Feldstudien stehen noch nicht zur Verfügung, ein Wechsel auf quadrivalente Impfstoffe sollte aber aufgrund des Spektrums und der betroffenen Serovare trotzdem empfohlen werden. Eine erneute Grundimmunisierung mit 2 Injektionen innerhalb von 3-4 Wochen mit dem neuen Impfstoff ist essenziell für einen ausreichenden Schutz gegen die neuen Valenzen.

Online zu finden unter

http://dx.doi.org/10.1055/s-0033-1361519

\section{Literatur}

1 Barmettler R, Schweighauser A, Bigler S et al. Assessment of exposure to Leptospira serovars in veterinary staff and dog owners in contact with infected dogs. J Am Vet Med Assoc 2011; 238: 183-188

2 Geigy CA, Schweighauser A, Doherr M et al. Occurrence of systemic hypertension in dogs with acute kidney injury and treatment with amlodipine besylate. I Small Anim Pract 2011; 52: 340-346

3 Geisen V, Stengel C, Brem S et al. Canine leptospirosis infections - clinical signs and outcome with different suspected Leptospira serogroups (42 cases). J Small Anim Pract 2007; 48: 324-328

4 Geisen V, Stengel C, Hartmann K. Epidemiologic situation of leptospirosis in dogs in the Southern states of Germany. Tierärztl Praxis, Kleintiere 2008; 36: 329-336

5 Gerlach T, Stephan I. Epidemiologic situation of canine leptospirosis in the Northern states of Germany in the years 2003-2006. A retrospective study. Tierärztl Praxis Kleintiere 2007; 35: 123-128

6 Goldstein RE. Canine leptospirosis. Vet Clin North Am Small Anim Pract 2010; 40: 1091-1101
7 Hartmann K, Stützer B. Leptospirose beim Hund - Überblick über Bedeutung, Klinik und Prophylaxe. Veterinär Spiegel 2012; 22: 51-54

8 Kis I et al. Long-term renal outcome of dogs with acute kidney injury [Abstract]. J Vet Intern Med 2012; 26

9 Kohn B, Steinicke K, Arndt G et al. Pulmonary abnormalities in dogs with leptospirosis. J Vet Intern Med 2010; 24: 1277-1282

10 Schweighauser A et al. Small intestinal intussusception in five dogs with acute renal failure and suspected leptospirosis (L. australis). J Vet Emerg Crit Care 2009; 19: 363-368

11 Sykes JE, Hartmann K, Lunn KF et al. 2010 ACVIM Small animal consensus statement on leptospirosis: diagnosis, epidemiology, treatment, and prevention. J Vet Intern Med 2011; 25: 1-13

Dr. med. vet. Thierry Francey, Dipl. ACVIM (SAIM)

Dr. med. vet. Ariane Schweighauser, Dipl. ACVIM (SAIM)

Vetsuisse Fakultät Universität Bern

Länggassstraße 128, 3001 Bern, Schweiz 


\section{Fragebogen}

\section{Frage 1}

Welche der folgenden Tierarten wird in manchen Ländern wegen ihrer möglichen Rolle in der Übertragung der Leptospirose seuchenpolizeilich überwacht?
a. die Ratte
b. das Reh
c. die Kuh
d. der Fuchs
e. der Dachs

\section{Frage 2}

\section{Was ist der häufigste natürliche} Infektionsweg für die Leptospirose des Hundes?

a. ein direkter Kontakt mit Trägertieren

b. eine iatrogene Übertragung bei Bluttransfusion

c. eine transplazentare Übertragung

d. eine Übertragung über Muttermilch

e. eine indirekte Übertragung über

kontaminierte stehende Gewässer

\section{Frage 3}

Was ist keine typische klinische Manifestation der Leptospirose beim Hund?

a. akutes Nierenversagen

b. hämolytische Anämie

c. Hepatopathie

d. Lungenblutungen

e. Gerinnungsstörungen

\section{Frage 4}

Welches Nierenkompartiment ist am stärksten betroffen bei der Hundeleptospirose?
a. die Nierenkapsel
b. das Nierenbecken
c. das Gefäßsystem
d. der Glomerulus
e. das Tubulointerstitium

\section{Frage 5}

Welche der folgenden Aussagen über die Diagnosestellung der Leptospirose mittels MAT-Serologie trifft zu?

a. Die MAT-Serologie dient dem Nachweis von Leptospiren-Antigenen im Serum.

b. Eine negative MAT-Serologie bei der initialen Vorstellung schließt eine Leptospirose aus.

c. Die MAT-Serologie erlaubt die Differenzierung zwischen Impfung und Infektion-induzierter Serokonversion.

d. Gepaarte MAT-Titer werden in der Regel als Goldstandard betrachtet.

e. Eine MAT-Serokonversion identifiziert eindeutig das infizierende Serovar.

\section{Frage 6}

Welche der folgenden Aussagen betreffend die PCR-Diagnose für Leptospirose trifft zu?

a. Der Nachweis von Proteinen der Leptospiren mittels PCR kann aus dem Blut erfolgen.

b. PCR aus Urin gilt als Goldstandard für Leptospirose und ein positives Resultat ist eindeutig beweisend für eine aktive Infektion.

c. Eine Therapie mit Antibiotika führt rasch zu einer negativen PCR aus Blut und Urin.

d. Leptospiren-DNA verschwindet schneller aus Nierengewebe als aus Blut.

e. Die PCR ist eine gute Methode zur Identifizierung des kausalen Leptospiren-Stamms.

\section{Frage 7}

Welches der folgenden Antibiotika ist beim Verdacht auf Leptospirose bei einem Hund mit akutem schweren Erbrechen und Durchfall am besten geeignet?
a. Penicillin G
b. Doxycyclin
c. Enrofloxacin
d. Cefazolin
e. keines - es wird empfohlen, die Resultate der Serologie und die Bestätigung der Diagnose abzuwarten

\section{Frage 8}

Welche der folgenden Aussagen betreffend Flüssigkeitstherapie trifft am ehesten zu?

a. Jedes Tier mit akutem Nierenversagen braucht Flüssigkeitstherapie.

b. Überhydratation und Hypervolämie sind bei oligurischem akutem Nierenversagen unvermeidbar.

c. Kolloide sind bei Leptospirose besser geeignet als Kristalloide.

d. Die Flüssigkeitstherapie muss individuell verschrieben werden.

e. Hunde mit Leptospirose sollten mit 2facher Erhaltung Kristalloide behandelt werden.

\section{Frage 9}

Welches der folgenden Medikamente hat keine Indikation bei der Behandlung der akuten Hundeleptospirose?

a. Benazepril

b. Metoclopramid

c. Amlodipin

d. Buprenorphin

e. Maropitant

\section{Frage 10}

Welche Aussage zur Prophylaxe der Leptospirose beim Hund trifft am ehesten zu?

a. Alte bivalente Impfstoffe sind wegen ihrer mit älteren Methoden hergestellten Adjuvanzien obsolet.

b. Neue quadrivalente Impfstoffe decken alle aktuellen Serovare ab.

c. Eine neue Grundimmunisierung mit 2 facher Impfung ist beim Wechsel von einem bivalenten auf einen quadrivalenten Impfstoff nötig.

d. Großflächige Feldstudien haben die Wirksamkeit der neuen quadrivalenten Impfstoffe in unseren Breitengraden eindeutig bewiesen.

e. Die neuen quadrivalenten Impfstoffe beinhalten nicht die aktuell betroffenen Serovare; sie sollten aber durch Kreuzreaktion genügend Immunität hervorrufen. 


\section{Leptospirose beim Hund - ein Update}

Thierry Francey, Ariane Schweighauser

A Lernerfolgskontrolle

\section{Bitte kreuzen Sie die richtigen Antworten an! Es ist jeweils nur 1 Antwort pro Frage richtig!}

\section{B Teilnehmer}

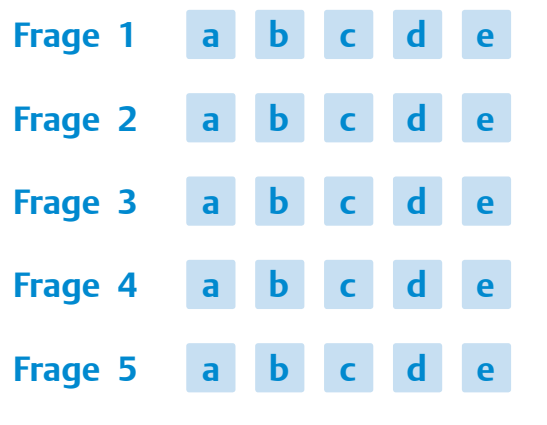

\section{Frage 6}

Frage 7

Frage 8

Frage 9

Frage 10

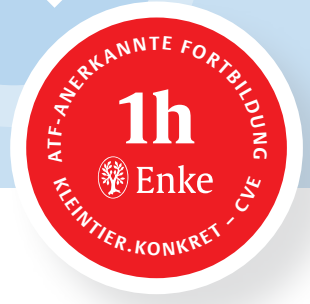

Beruf

\section{Ihr Ergebnis wird vom Verlag ausgefültt}

Sie haben von $\square \quad$ Fragen bestanden und 1 ATF-Stunde erhalten.

richtig beantwortet und somit

Stuttgart, den

\section{Teilnahmebedingungen für Abonnenten der kleintier konkret kostenlos}

\section{E Erklärung}

Ich versichere, dass ich die Beantwortung der Fragen selbst und ohne Hilfe durchgeführt habe.

Für diese Fortbildungseinheit können Sie 1 ATFFortbildungsstunde anerkannt bekommen. Hierfür beantwortet sein.

muss der Antwortbogen vollständig ausgefüllt sein. Unvollständig ausgefüllte Bögen können nicht berücksichtigt werden!

Ort, Datum
- müssen mindestens $70 \%$ der Fragen richtig

\section{muss im markierten Feld* Ihre Abonnen- tennummer eingetragen oder eine kleintier konkret-CVE-Wertmarke aufgeklebt sein.}

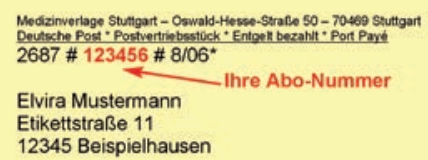

kleintier konkret-CVE-Wertmarken für NichtAbonnenten können beim Verlag zu folgenden Bedingungen erworben werden: 6erPackWertmarken, Preis 49,95€ inkl. MWSt., Artikel-Nr. 903000.

Bitte richten Sie die Bestellungen an: MVS Medizinverlage Stuttgart,

KundenServiceCenter Buch, Postfach 3011 20, 70451 Stuttgart.

* Nicht-Abonnenten bitte hier kleintier konkret-CVE-Wertmarke aufkleben, Abonnenten bitte Abonnentennummer eintragen 